OPEN ACCESS

Edited by:

Peter Poczai,

University of Helsinki, Finland

Reviewed by:

Abdullah,

Quaid-i-Azam University, Pakistan

Ibrar Ahmed,

Alpha Genomics Private Limited,

Pakistan

*Correspondence:

José M. Gualberto

jose.gualberto@ibmp-cnrs.unistra.fr

Specialty section:

This article was submitted to Plant Systematics and Evolution,

a section of the journal

Frontiers in Plant Science

Received: 18 April 2021 Accepted: 05 July 2021

Published: 26 July 2021

Citation:

Fertet $A$, Graindorge $S$, Koechler S, de Boer G-J, Guilloteau-Fonteny E and

Gualberto JM (2021) Sequence of the Mitochondrial Genome of Lactuca virosa Suggests an Unexpected Role

in Lactuca sativa's Evolution.

Front. Plant Sci. 12:697136. doi: 10.3389/fp/s.2021.697136

\section{Sequence of the Mitochondrial Genome of Lactuca virosa Suggests an Unexpected Role in Lactuca sativa's Evolution}

\author{
Arnaud Fertet ${ }^{1}$, Stéfanie Graindorge ${ }^{1}$, Sandrine Koechler ${ }^{1}$, Gert-Jan de Boer ${ }^{2}$, \\ Emilie Guilloteau-Fonteny ${ }^{3}$ and José M. Gualberto ${ }^{1 *}$
}

${ }^{1}$ Institut de Biologie Moléculaire des Plantes, CNRS, Université de Strasbourg, Strasbourg, France, ${ }^{2}$ Enza Zaden Research and Development B.V., Enkhuizen, Netherlands, ${ }^{3}$ Enza Zaden France Recherche S.A.S., Allonnes, France

The involvement of the different Lactuca species in the domestication and diversification of cultivated lettuce is not totally understood. Lactuca serriola is considered as the direct ancestor and the closest relative to Lactuca sativa, while the other wild species that can be crossed with L. sativa, Lactuca virosa, and Lactuca saligna, would have just contributed to the latter diversification of cultivated typologies. To contribute to the study of Lactuca evolution, we assembled the mtDNA genomes of nine Lactuca spp. accessions, among them three from $L$. virosa, whose mtDNA had not been studied so far. Our results unveiled little to no intraspecies variation among Lactuca species, with the exception of $L$. serriola where the accessions we sequenced diverge significantly from the mtDNA of a $L$. serriola accession already reported. Furthermore, we found a remarkable phylogenetic closeness between the mtDNA of $L$. sativa and the mtDNA of $L$. virosa, contrasting to the $L$. serriola origin of the nuclear and plastidial genomes. These results suggest that a cross between $L$. virosa and the ancestor of cultivated lettuce is at the origin of the actual mitochondrial genome of $L$. sativa.

Keywords: mitochondrial genome, genome evolution, lettuce, Lactuca virosa, Lactuca serriola, Lactuca saligna

\section{INTRODUCTION}

Within the Lactuca genus which contains about 100 species, four species are well-described and characterized: the cultivated one, Lactuca sativa, and three wild species, L. saligna, L. serriola, and Lactuca virosa, which can all be crossed with L. sativa (Ryder, 1999). L. saligna, with its long and narrow leaves (Lindqvist, 1960), is also called "least lettuce," while L. serriola is named "prickly lettuce," because of the harder prickles in its lobed leaves and stem. L. virosa, or "great lettuce," shows many phenotypic variations according to ecotypes: leaves can be lobed or not, some with prickles others not, but all have broad leaves (Lindqvist, 1960). The differences between L. sativa, the domesticated lettuce, and the wild species are morphological, like the formation of the lettuce head at the vegetative stage, and a less bitter taste through the reduction of latex production. On the market, four L. sativa typologies are predominant (Davis et al., 1997): Crisphead lettuces (var. capitata L. salinas), Cos lettuces (var. longifolia), leaf lettuces (var. acephala Alef.), and Butterhead lettuces (var. capitata L. nidus tenerrima). 
The process of domestication, that led to L. sativa, is not clearly understood. It is established that L. serriola is one of the direct ancestors and the closest related species (de Vries, 1990; Kesseli et al., 1991; de Vries and van Raamsdonk, 1994) and that hybrids L. sativa $\times$ L. serriola are self-fertile (Thompson et al., 1941; Thompson, 1943). L. virosa and L. saligna are playing an important role in the development of lettuce cultivars, and especially in the introgression of resistance genes like the ones for Bremia lactucae (Parra et al., 2021). However, a study based on RFLP markers suggested that L. sativa could have a polyphyletic origin, but without being able to identify other contributing species (Kesseli et al., 1991). More recently, a study investigated the genealogy of North American lettuces and identified L. virosa as introgressed in L. sativa in certain lineages of cultivars, such as in a group of Crisphead lettuces. L. virosa would contribute to a more robust root system and decreased leaf drop (Mikel, 2007). The advances in sequencing technologies and the recent increase in available sequenced genomes helped to clarify the relationship between Lactuca spp. Two sets of markers were developed: as nuclear marker, a set of ribosomal internal transcribed spacers (ITS), ITS-1 and ITS-2, which flank the 5.8 rRNA gene sequence, and as chloroplast marker the concatenated sequence of the $n d h F$ and $\operatorname{trnL}-F$ genes (Koopman et al., 1998; Wei et al., 2017). These studies showed that the Lactuca spp. can be subdivided into several clades among which the crop group containing L. sativa, L. saligna, L. serriola, and L. virosa (Wei et al., 2017). This subdivision goes further and the species can be distinguished, with the very exception of L. sativa and L. serriola that could not be separated from each other (Koopman et al., 2001). Regarding the mitochondrial genome (or mtDNA), the ones of L. sativa (363 kb), L. saligna (368 kb), and L. serriola (363 kb) were recently published, which allowed the development of a new set of markers (Kozik et al., 2019).

It is not surprising that the last $L$. sativa genome released was the mtDNA. Indeed, the large size and complex and dynamic structure of plant mitochondrial genomes makes their sequencing and assembly delicate. The presence of mitochondrial sequences inserted in the nuclear genome, also known as NUMTs (Nuclear MiTochondrial sequences), further complicates their assembly from total genomic data. This is a relatively lesser issue in Arabidopsis thaliana and its small nuclear genome of $\pm 157 \mathrm{Mb}$ (The Arabidopsis Genome Initiative, 2000; Bennett et al., 2003), but it is a different situation for plants with much bigger nuclear genomes, such as lettuce (2.9 Gb) (Reyes-Chin-Wo et al., 2017). There are two ways to work around this problem: choose a higher-throughput sequencing strategy to increase sequencing deep, or enrich the mtDNA concentration by extracting it from purified mitochondria. Then, once the sequencing is done the challenge is not finished. Plant mitochondrial genomes have highly dynamic and multipartite structures because of large repeated sequences ( $\geq 500 \mathrm{bp}$ ) involved in frequent and reciprocal recombination, which makes their assembly tricky. These large repeated regions cannot be crossed by a conventional short-read sequencing strategy. Consequently, during assembly these sequences remain contigs that can be linked to four other contigs (two per side) leading to four possible configurations. This assembly issue can only be overcome using a long-reads strategy (Pacbio SMRT or Illumina mate-pair) in combination with a short-reads one.

The content of plant mtDNAs varies little between species. Indeed, there are rarely any differences in gene content within a genus. Even in Silene spp. where there are massive changes in mtDNA genome size and structure, very few differences in gene content can be observed (Sloan et al., 2012). And there are even fewer intraspecific differences, like in A. thaliana, where different accessions have the same content in protein and tRNA genes (Davila et al., 2011; Sloan et al., 2018). In fact, the diversity of plant mtDNAs is mainly found in their structure, such as in $A$. thaliana where the different sequences of the mtDNA are differently organized in different accessions, as in Col-0, C24, and Landsberg erecta (Davila et al., 2011). A major source of diversity in plant mtDNA content comes from its interaction with other genomes, by horizontal sequence transfer (Marienfeld et al., 1999; Bergthorsson et al., 2003), and in particular with the plastidial genome (cpDNA). Indeed, the mtDNA of plants is often strewn with plastid DNA insertions. Most plastid DNA sequences transferred to the mtDNA are neutral (Cummings et al., 2003), but several chloroplast-derived tRNA genes have been recruited to be functional in mitochondria (Dietrich et al., 1996).

In this work our goal was to assess the diversity of mtDNA genomes interspecies among different Lactuca species, and intraspecies among different accessions of the same species. The final objective is to better understand the contribution of the different wild-type Lactuca species to the domestication and diversification of L. sativa. Here we determined the complete mtDNA sequences of nine accessions from four related species in the genus Lactuca. The nuclear data confirmed previous reports showing that L. sativa and L. serriola are closely related species and distinct from L. saligna and L. virosa (Koopman et al., 1998; Wei et al., 2017; Zhang et al., 2017). The phylogeny of the complete chloroplastic genome sequences was in line with the nuclear one. However, we found that from the sequence and structural points of view the mtDNA of $L$. sativa is closer to the one from L. virosa than to the ones from L. saligna or L. serriola. The close phylogenetic relation between the mtDNA of L. sativa and $L$. virosa is further supported by a clear divergence in cpDNA insertions in the mtDNA, where L. sativa and L. virosa share a same specific one, whereas $L$. serriola and L. saligna share a distinct cpDNA insertion. Our results highlight a complex origin of the organellar genomes of cultivated lettuce.

\section{MATERIALS AND METHODS}

\section{Plant Material and Growth Conditions}

A collection of nine accessions (Supplementary Table 1) belonging to four different Lactuca species (L. sativa, L. saligna, L. serriola, and L. virosa) originating from diverse geographical origins were used in this study and seeds are available upon request. Seeds of all accessions were provided by the company Enza Zaden (Enkhuizen, Netherlands), and propagated to obtain sufficient seeds for mitochondria purification. All accessions are recorded in the company Genbank and were isolated either by Enza Zaden (L. sativa accessions LACWENDEL and 
LAC004500, L. saligna accession LAC008020, L. serriola accession LAC005780, L. virosa accession LAC006941) or originate from other collections ( $L$. serriola accession CGN004799, L. virosa accessions CGN013357 and CGN019045 are from the Centre for Genetic Resources, Wageningen University and Research and L. sativa accession UC12100 is from the University of California, Davis).

Seeds were sterilized prior to growth by incubation for $30 \mathrm{~s}$ with $70 \%$ ethanol followed by 10 min with $1 \% \mathrm{NaOH}$, washed with sterile water and dried on blotting paper in a sterile flow cabinet. Sterilized seeds were placed on T-300 blotting paper $(\varnothing$ 80 mm; All Paper b.v., Didam, Netherlands) in Pétri dishes and wet with $5 \mathrm{~mL}$ of sterile water. The Pétri dishes were sealed with parafilm to keep humidity and stratified for 2 days at $4^{\circ} \mathrm{C}$ to induce germination, followed by growth in the dark at $21^{\circ} \mathrm{C}$ up to 7 days.

\section{Mitochondrial DNA Extraction}

Whole 7 days old Lactuca etiolated seedlings were used for mitochondria preparation. Briefly, about 1,000 seedlings are ground with a mortar and pestle in grinding buffer $(0.6 \mathrm{M}$ sucrose, $50 \mathrm{mM}$ tetrasodium pyrophosphate, $4 \mathrm{mM}$ EDTA, $20 \mathrm{mM}$ KH2PO4, 2\% PVP-40, 2\% BSA, pH 7.5, $40 \mathrm{mM}$ ascorbate, $10 \mathrm{mM}$ cysteine, $2 \mathrm{mM}$ DTT; $10 \mathrm{~mL}$ for 100 seedlings). The suspension is filtered through two layers of nylon mesh (96 $\mu \mathrm{m}$ ) and the solid material is ground a second time in $10 \mathrm{~mL}$ of grinding buffer and filtered. The suspension is then fractionated by differential centrifugation to eliminate particles with low sedimentation coefficients $(10 \mathrm{~min}$ at $3,500 \mathrm{~g}$, twice $5 \mathrm{~min}$ at $3,500 \mathrm{~g}, 5 \mathrm{~min}$ at $6,000 \mathrm{~g}$ ) followed by a final sedimentation of the mitochondria enriched fraction $(20 \mathrm{~min}$ at $17,000 \mathrm{~g})$. The pellet is then resuspended in washing buffer (0.3 M sucrose, $10 \mathrm{mM}$ MOPS, 0.1\% BSA, pH 7.5) and mitochondria purified on Percoll step gradients (18-25-50\% representing 33-50-17\% of the total gradient volume of $2.2 \mathrm{~mL}$ ), at 25,000 $\mathrm{g}$ for $25 \mathrm{~min}$ on an Optima MAX-TL (rotor TLA 100.3) from Beckman Coulter (Brea, CA, United States). The purified mitochondria band is collected at the $25-50 \%$ Percoll interphase. After mitochondria purification, contaminant nuclear or plastid DNA bound to the mitochondria surface is removed by DNase treatment. For that mitochondria are resuspended in $100 \mu \mathrm{L}$ of washing buffer containing $50 \mu \mathrm{g}$ DNase I and $0.55 \mu \mathrm{M}$ $\mathrm{Mg} 2+$ and incubated $20 \mathrm{~min}$ at $25^{\circ} \mathrm{C}$. After incubation, $1 \mathrm{~mL}$ of washing medium containing EDTA and EGTA (both at $10 \mathrm{mM}$ ) is added and mitochondria sedimented at $18,000 \mathrm{~g}$ for $15 \mathrm{~min}$. DNA extraction from purified mitochondria was performed using the QIAamp ${ }^{\circledR}$ DNA Micro kit (Qiagen, Venlo, Netherlands) following the protocol "Isolation of Genomic DNA from tissues."

\section{DNA Sequencing}

For Illumina sequencing, library preparation and sequencing reactions were performed by the IBMP "gene expression analysis" platform. Libraries were prepared with the Nextera XT DNA Library Prep kit (Illumina, San Diego, CA, United States) and their size checked on a 2100 Bioanalyzer (Agilent, Santa Clara, CA, United States). The libraries were then sequenced with paired-end $2 \times 151$ reads on a MiSeq System (Illumina). The raw Illumina reads were deposited in GenBank under accession numbers ERS5266978 and ERS5267070 to ERS5267078. ${ }^{1}$ Pacbio sequencing of the mtDNA of $L$. sativa var. capitata L. nidus tenerrima (accession LACWendel) was performed by Keygene (Wageningen, Netherlands) on a Pacbio Sequel II instrument (Pacific Biosciences, Menlo Park, CA, United States). The sequencing generated $19.9 \mathrm{~Gb}$ from 3.2 million reads (average read size 3,051 pb). The raw PacBio reads were deposited in GenBank under accession number ERS5266954. ${ }^{1}$

\section{Sequences Alignments and Phylogenetic Analysis}

From GenBank we obtained 88 Lactuca ITS1-5.8S-ITS2 sequences from eight species: L. sativa, Lactuca dregeana, Lactuca indica, L. saligna, Lactuca scandens, L. serriola, Lactuca sibirica, and Lactuca virosa (Supplementary Table 2). For the ITS1-5.8S-ITS2 sequences of accessions described in Supplementary Table 1 and from Kozik et al. (2019) the Illumina reads were mapped on L. sativa references (KT249801.1) with the Burrows-Wheeler Aligner bwa-mem (v07.7.17-r1188) (Li and Durbin, 2009) and mapped reads were assembled with SPAdes (v3.13.1) (Bankevich et al., 2012). Finally, assembled sequences were manually trimmed to match starts and ends of reference sequences. Sequence alignments were performed on MEGA $X$ (v10.1.7) (Kumar et al., 2018; Stecher et al., 2020) using MUSCLE (Edgar, 2004) with default options. Maximum likelihood trees were also built on MEGA $X$, using the bootstrap method (1,000 replications) with default options and final trees were computed on the Interactive Tree Of Life (v6) (Letunic and Bork, 2019).

The plastid genomes assembly and comparison was performed using MacVector (v18.1.5) (MacVector INC., Apex, NC, United States) Assembler plugin's. Illumina reads were mapped on the L. sativa cultivar Salinas cpDNA reference (AP007232.1) with the aligner Bowtie v1.3.0 (Langmead et al., 2009). Sequences were aligned with ClustalW 1.83 implemented in the MacVector package using default options. Regions of ambiguity that are duplicated in the mitochondrial genome were removed from the alignment. Maximum likelihood trees were built by the neighbor-joining method. The alignment is given as Supplementary Data.

Concatenated protein coding sequences of the mtDNA were manually assembled, from the annotations of the genomes. They were aligned with ClustalW 1.83 and maximum likelihood trees built by the neighbor-joining method, using the software implemented in the MacVector package. The alignment is given as Supplementary Data.

\section{Assembly of the Mitochondrial Genomes}

The assembly of the mtDNA of $L$. sativa var. capitata $L$. nidus tenerrima combined Illumina and Pacbio reads. Contigs from Illumina MiSeq paired-end $(2 \times 150$ bases $)$ were built with the organellar de novo assembler Novoplasty (v2.4) ( $k$ mer 22) (Dierckxsens et al., 2016). This led to 48 contigs, among them 22 were confirmed as mtDNA contigs by BLAST

\footnotetext{
${ }^{1}$ www.ebi.ac.uk/ena
} 
(Altschul et al., 1990). Repeated regions were identified as the overlap between four different contigs and particularly three large ones: R01 (34,696 bp), R02 (10,430 bp), and R03 (3,552 bp). Raw Pacbio data delivered by Keygene were mapped on the $21 \mathrm{mtDNA}$ contigs from Illumina with bwamem (Supplementary Figure 1A). Mapped Pacbio reads were then assembled using Canu (Koren et al., 2017) to obtain four contigs. Among them three large ones were identified as mitochondrial by BLAST (v2.10.1) (Altschul et al., 1990): 177,077 bp (PB01), 90,502 bp (PB02), and 73,475 bp (PB03). Interestingly, the start of the $\mathrm{PB} 01$ sequence overlaps its end for 5,225 bp. Consequently, we circularized it into a circular chromosome (PBc01) of 171,852 bp. Furthermore, we found that the extremities of contigs PB02 and PB03 mapped to sequences internal to $\mathrm{PBc} 01$, corresponding to repeated regions identified among Novoplasty contigs. So we circularized PB02 and PB03 using repeats R01 and R02, respectively, into circular chromosomes $\mathrm{PBc} 02$ (112,968 bp) and $\mathrm{PBc} 03$ (78,504 bp). The circular chromosomes were then assembled through recombination-like events involving repeated sequences R01 and R02 into a single circular molecule of $363,324 \mathrm{bp}$. The code used for the assembly is available at github.com/ARNTET/Pacbio_Illumina_mtDNA_assembly.

The assembly of the L. saligna, L. sativa, L. serriola, and L. virosa mitochondrial genomes was from Illumina reads. We built our pipeline (Supplementary Figure 1B) based on a recent work from Garcia et al. (2019). Illumina reads from Lactuca species were trimmed with Trimmomatic (v0.39) (Bolger et al., 2014) to remove adapter sequences and low quality ends and joined with Fastq-join (v1.3.1) ${ }^{2}$ when paired reads mapped to each other. Then those reads were assembled into contigs with a first assembler: Velvet (v1.2.10) (Zerbino and Birney, 2008). Those contigs were mapped on the L. sativa var. capitata L. nidus tenerrima mtDNA sequence and mapped Velvet contigs were used as trusted contigs for a second round of assembly with SPAdes (v13.3.1) (Bankevich et al., 2012). SPAdes contigs were identified by BLAST (v2.10.1) (Altschul et al., 1990) and filtered according to their coverage. Filtered SPAdes contigs were extended with SSPACE (v1.12) into long scaffolds (up $170 \mathrm{~kb}$ ). Finally, contigs were manually assembled into single circular molecules. The code used for the assembly is available at github.com/ARNTET/Illumina_plant_mtDNA_assembly.

\section{Annotation of the Genomes}

Lactuca sativa mtDNA was first automatically annotated using GeSeq (v2.0.3) (Tillich et al., 2017) with default settings for circular mitochondrial genome and with Diplostephium hartwegii (KX063855.1) as 3rd party reference. Then the annotation was manually reviewed with $L$. sativa RNA-seq data (accessions SRR080725 and SRR085107). RNA-seq reads were mapped on L. sativa mtDNA using Hisat2 (v2.1.0) (Kim et al., 2015) with reduced mismatch penalties (-mp parameter to " 2.0 ”), allowing the mapping of more reads. SAM format files were then converted to BAM format with Samtools (v1.9) (Li et al., 2009). BAM files were sorted with Samtools for visualization on $I G V$ (v2.8.12)

${ }^{2}$ http://github.com/ExpressionAnalysis/ea-utils
(Robinson et al., 2011) to review CDS border. SNPs calling was made with Freebayes (v1.3.1) (Garrison and Marth, 2012), using default parameters. SNPs were filtered to remove non-C-to- $\mathrm{U}$ (or G-to-A) ones. Finally, only SNPs in coding regions were conserved and annotated as RNA editing sites. Code available at github.com/ARNTET/RNAseq_mapping_hisat2.

The L. saligna, L. serriola, and L. virosa mitochondrial genomes were automatically annotated using GeSeq (v2.0.3) (Tillich et al., 2017) with default settings for circular mitochondrial genome and with the $L$. sativa mtDNA and the recently published $L$. serriola US96UC23 and L. saligna CGN5271 mtDNAs (MK820672.1 and MK759657.1, respectively) as "3rd party references." The repeated regions among the Lactuca mtDNA sequences were identified by using the python script ROUSfinder (v1.1) (Wynn and Christensen, 2019). We annotated the sequences larger than 50 bp from the largest (R01) to the smallest (R17). The nomenclature in our dataset is based on the one of L. sativa, homologous repeated regions in the others Lactuca species mitogenomes are annotated with the same number. Visualization and identification of blocks of synteny between the mtDNA genomes assembled in this work was done with progressiveMauve (v2.4.0) (Darling et al., 2010) with default options. The annotated mtDNA sequences have been deposited in GenBank under accession numbers MZ159953 (LsatUC12100), MZ159954 (LsatLACWENDEL), MZ159955 (LsatLAC004500), MZ159956 (LsalLAC008020), MZ159957 (LserCGN004799), MZ159958 (LserLAC005780), MZ159959 (LvirLAC006941), MZ159960 (LvirCGN013357), and MZ159961 (LvirCGN019045).

\section{RESULTS}

\section{Phenotypic Diversity Within a Collection of Nine Accessions of Cultivated and Wild Lettuce}

For this study, we selected nine accessions of Lactuca spp. among those available at the Genbank of Enza Zaden (Enkhuizen, Netherlands): three of $L$. sativa, one of $L$. saligna, two of L. serriola, and three of $L$. virosa. The three L. sativa accessions represent two cultivars: LACWendel is a Butterhead lettuce (L. sativa var. capitata L. nidus tenerrima), and UC12100 and LAC004500 are Crisphead lettuce (var. capitata L. salinas). The accessions were selected to include the three wild Lactuca species believed to be at the origin of domesticated $L$. sativa, and covering a wide diversity of geographical origins (Supplementary Table 1). Accessions showed marked phenotypical variation according to the species, but also interspecies (Figure 1). At the seed level, seeds from $L$. virosa accessions are large and black, while L. saligna and L. serriola seeds are thinner and brown. The L. sativa LAC011481 seeds are also thin and brown, while those of L. sativa LACWendel and LAC004500 are white. The phenotypic variations are even stronger at the rosette stage. L. sativa accessions show upright spooned leaves while the leaves of the L. virosa accessions are long and droopy. The leaves 

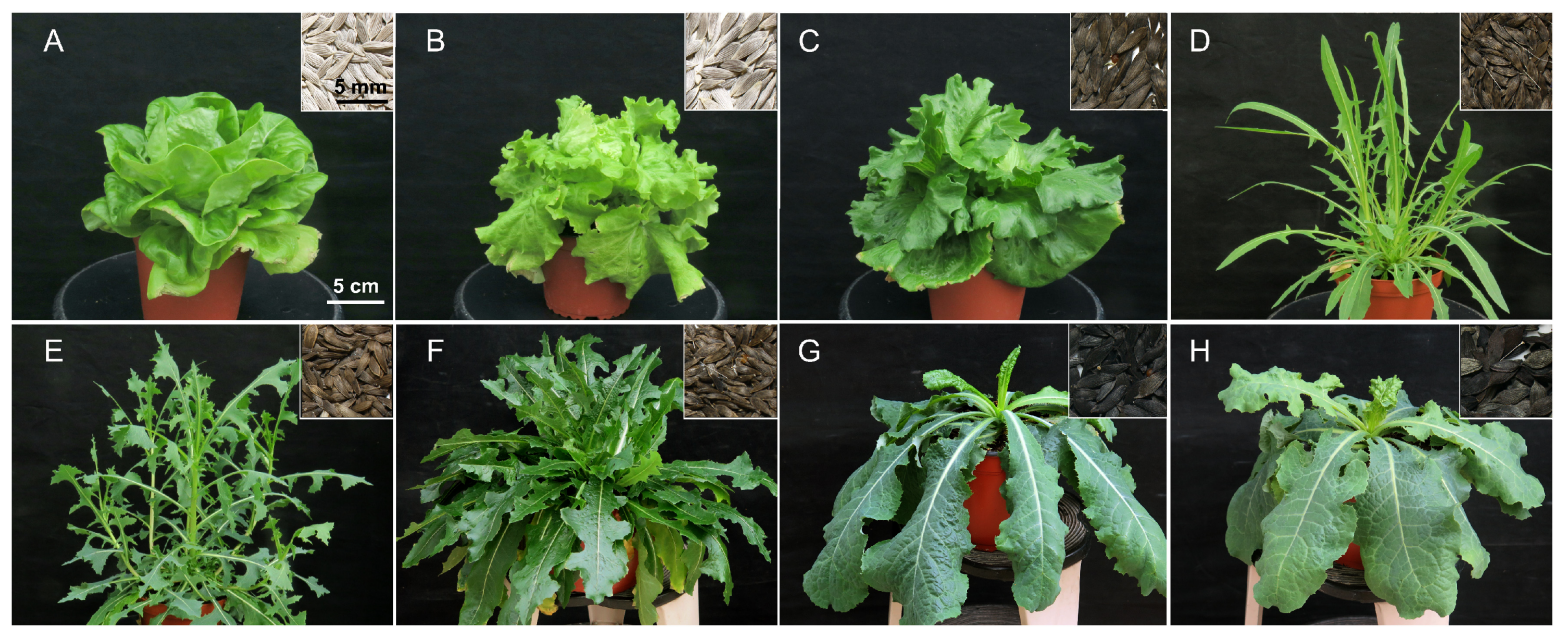

FIGURE 1 | Phenotypes of the selected Lactuca at the rosette stage and corresponding seeds. (A) L. sativa LACWendel; (B) L. sativa LAC004500; (C) L. sativa US12100; (D) L. saligna LAC008020; (E) L. serriola CGN004799; (F) L. serriola LAC005780; (G) L. virosa CGN013357; (H) L. virosa LAC006941. L. virosa CGN019045 is not represented but it is similar to the two others $L$. virosa accessions, in terms of leaves and seeds shape and color.

of $L$. serriola plants are lobed shaped, but the ones L. saligna LAC00820 are upright needle-like.

\section{Nuclear Sequence Diversity Among Accessions}

We propagated the seeds of the selected accessions and prepared DNA from purified mitochondria, as described in "Materials and Methods." The enrichment in mitochondrial DNA as compared to nuclear and plastidial DNA was evaluated by $\mathrm{qPCR}$, using primer pairs specific for each genomic compartment: $18 S$ nuclear rRNA gene, $18 S$ mitochondrial rRNA gene, NAD3 mitochondrial gene and RBCL plastid gene (Supplementary Table 3). For all samples the mtDNA was enriched more than 100-fold (up to $\sim 10,000$-fold for LACWendel), except for L. virosa LAC006941 ( $\sim 80$-fold), while the cpDNA was poorly enriched $(\sim 2.5$ to $\sim 20$-fold). We successfully sequenced the mtDNA enriched samples from the nine selected accessions by Illumina MiSeq $(2 \times 151 \mathrm{bp})$. These Illumina reads were evaluated afterward with the corresponding mtDNA built bellow, and showed a proportion of mitochondrial reads from $57 \%$ for $L$. sativa UC12100 to $5 \%$ for L. virosa LAC006941, allowing coverages of $1362 \mathrm{X}$ to 86X, respectively (Supplementary Table 4). We also obtained Pacbio long reads from L. sativa LACWENDEL. Our aim was to build the sequence and structure of the mtDNA of the corresponding accession, which could be used later as scaffolds to build the structures of the other accessions. Because we lacked information on the accessions from our population, besides the name of the species and from where they had been collected, we first established the nuclear diversity within this population to confirm their identity. To do so we built the set of ribosomal ITS, ITS- 1 and ITS-2, flanking the nuclear 5.8 rRNA gene sequence (640 bp), which has been previously used as nuclear marker to build phylogenetic relationships among Asteraceae species (Koopman et al., 1998). We did it for our selected accessions, from our Illumina reads, and for the L. sativa and L. serriola accessions from which the mtDNA was recently published and from which sequence data is available (accessions SRR080725 and SRR085107). We aligned the corresponding sequences to the 89 additional ones of Asteraceae species available on the NCBI nucleotide database (Supplementary Table 2). The alignment of these 100 sequences was 723 nucleotides long, comprising 404 conserved positions. The polyphyletic tree built using Maximum Likelihood from this alignment is shown in Figure 2. In this tree, our sequences branched as expected, in the same clusters as others from the same species. It is possible to distinguish every individual species, at the exception of $L$. sativa and L. serriola, which are unresolvable with this marker, as previously described.

This result confirmed the species identity of our selected accessions, and that $L$. sativa and $L$. serriola are closely related species that branch together and quite apart from $L$. saligna and L. virosa, at least at the nuclear level. On the other hand, our preliminary analysis of the nuclear sequences revealed that two accessions previously selected and annotated as L. saligna proved to be $L$. sativa accessions instead, highlighting the importance of previously confirming the identity of accessions obtained from germplasm banks.

\section{Mitochondrial Genomes of the Selected Lactuca spp. Accessions}

We assembled the mtDNA of $L$. sativa LACWENDEL by combining Illumina MiSeq $(2 \times 151 \mathrm{bp})$ and Pacbio long reads, as described in "Materials and Methods." The mtDNA of L. sativa accessions LAC004500 and UC12100, of L. saligna LAC008020, of L. serriola accessions CGN004799 and LAC005780 and of L. virosa accessions CGN013357, CGN019045, and LAC006941 were assembled by using Illumina MiSeq $(2 \times 151 \mathrm{bp})$ data only, from highly purified mtDNA. The assemblies generated, in each case, single circular molecules that are depicted in linearized forms in Figure 3. 


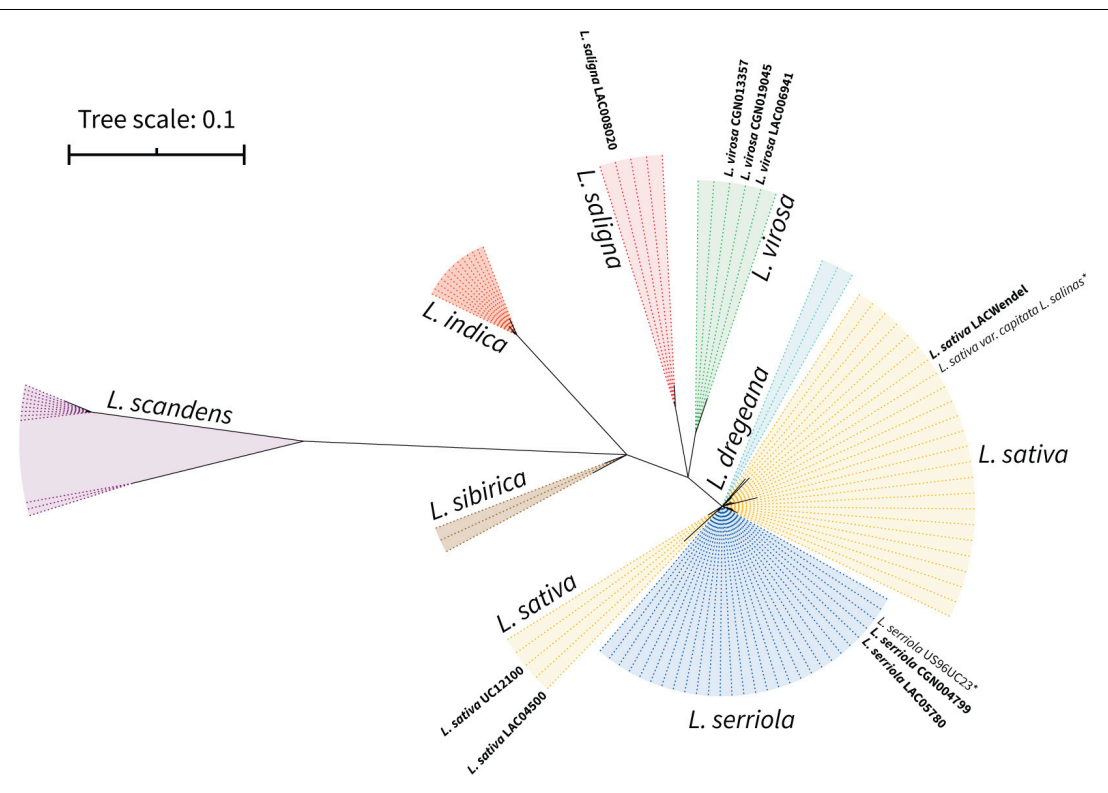

FIGURE 2 | Phylogenetic relation of the selected Lactuca accessions according to nuclear marker (ITS1-rRNA5.8S-ITS2). Maximum likelihood phylogram of the sequences of internal transcribed spacers 1 and 2 and the 5.8S rRNA. Bootstrap values are not shown but branches with BS <99 are collapsed. *Sequences assembled from Kozik et al. (2019) raw reads SRX5097892 (L. serriola US96UC23) and SRR577192 (L. sativa cv. Salinas).

The three L. sativa mtDNA genomes are 363,324 bp long and are $100 \%$ identical, and perfect isoforms of the recently published (Kozik et al., 2019) mtDNA of L. sativa cultivar Salinas (MK642355.1). The switch from one isoform to the other possible ones requires homologous recombination events involving the large repeated sequences $\mathrm{R} 02$ and R03, which are 10,430 and 3,553 bp long, respectively. A dynamic structure by recombination involving large repeats is a known and expected feature of plant mtDNA genomes. The mtDNA of L. saligna LAC008020 is 368,269 bp long. The structure that we obtained using our assembly pipeline is the perfect isoform to the recently published mtDNA of $L$. saligna CGN5271 (MK759657.1; Kozik et al., 2019). The two L. serriola mtDNAs that were assembled (accessions CGN004799 and LAC005780) are 367,647 bp long and identical, both in sequence and in structure. However, they diverge significantly from the recently published mtDNA of $L$. serriola accession US96UC23 (MK820672.1) which is 363,328 bp long (Kozik et al., 2019) and that seems to be an isoform of L. sativa mtDNA, with no sequence differences, while the mtDNAs of CGN004799 and LAC005780 are significantly different from L. sativa.

The mtDNAs of the three $L$. virosa accessions that were assembled (accessions CGN013357, CGN019045, and LAC006941) are 373,019 bp long. CGN013357 and LAC006941 are $100 \%$ identical and diverge from CGN019045 by two SNPs at positions 190,612 ( $\mathrm{C}$ to $\mathrm{A}$ ) and 190,615 ( $\mathrm{G}$ to $\mathrm{T}$ ). These positions are $\sim 600$ bp downstream nad7 in a non-coding region. Consequently, only one genome per species is represented in Figure 3.

These genomes are not only similar in size but also in gene content (Supplementary Tables 5, 6). Indeed, the mtDNAs of
L. sativa, L. saligna, L. serriola, and L. virosa all carry the same mitochondrial protein and rRNAs genes (Supplementary Table 6). The only difference in coding content concerns tRNA genes, more specifically one additional copy of the $\operatorname{trn} D$ (GUC) gene of plastidial origin which is found in the mtDNA of L. serriola. Mapping of available lettuce RNA-seq data (SRR080725 and SRR085107) confirmed expression of all protein and rRNA gene sequences. It also identified seven expressed orf s (orf15, orf43, orf47, orf52a, orf52b, orf163, and orf278) in the L. sativa mtDNA. Several of them seem to be co-transcribed with conventional mitochondrial genes; orf15 with cox2, orf 43 with cob, orf163 with nad5. The sequences and flanking regions of these orfs are conserved in all Lactuca mtDNAs that we built. Therefore, although there is no RNA-seq data for L. saligna, L. serriola, or L. virosa, these orfs are probably also transcribed in those species.

The RNA-seq data was also used to identify 738 RNA-editing sites in gene coding sequences. Among these sites, the one at position 245,933 (ACG) creates the initiation codon (AUG) of cox 1 , in $89 \%$ of the reads. The open reading frame of rpl16 overlaps with the $3^{\prime}$-end of rps3, like in all higher plant mtDNAs already sequenced (Newton et al., 1996). It was therefore believed that translation of $r p l 16$ is initiated internally to the rps3 sequence, but in a different frame (Takemura et al., 1992). However, it has been recently reported in Solanum tuberosum that an editing site that transforms an UCA serine codon into an UUA leucine codon in rps3 creates an internal UAG stop codon in the overlapping rpl16 reading frame, and that translation of rpl16 should initiate downstream of rps3 (Varré et al., 2019). We could identify the exact same event in L. sativa (editing site at position 151,176), thus confirming the strong conservation of this editing site and its essential importance for the correct 


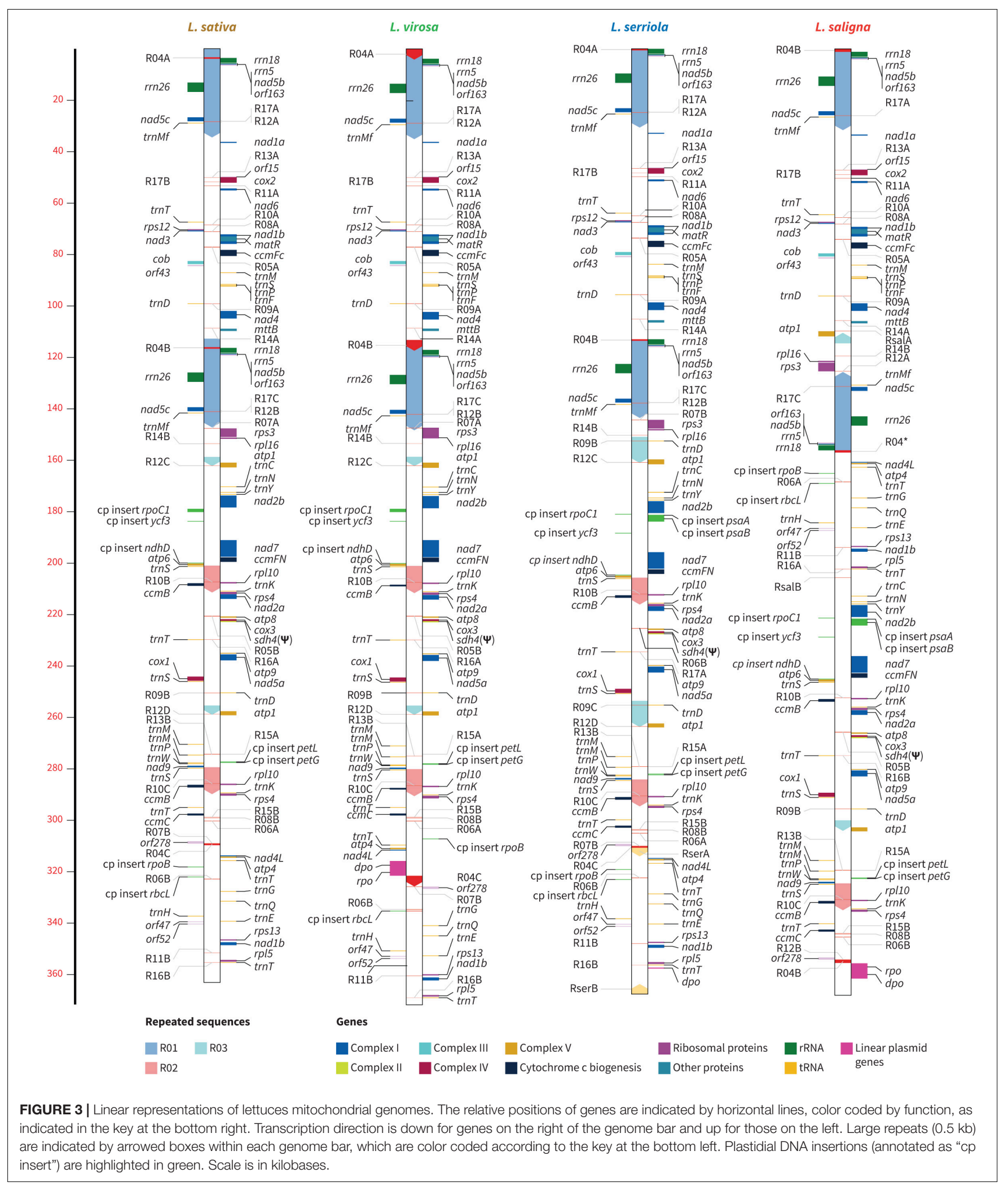

expression of rpl16. The recent determination of the structure of the plant mitochondrial ribosome (Waltz et al., 2020) showed that the mature rpl16 protein apparently starts at codon 37 of the corresponding orf, consistent with an initiation of translation at the GTG codon that is just upstream, as it was proposed (Varré et al., 2019). 
The same recent publication about the $S$. tuberosum mtDNA suggested methylation of the mitochondrial 18S rRNA (Varré et al., 2019). This hypothesis relies on RNA-seq data showing that in 55\% of reads a $\mathrm{T}$ is found at base 960, while an A is found in the corresponding genomic sequence. Such an event is diagnostic for the mis-incorporation of a $\mathrm{T}$ in front of an m1A nucleotide, during cDNA synthesis (Cognat et al., 2017). We observed the same event in L. sativa, where the A at position 961 of the 18S rRNA gene sequence (position 4,757 in the mtDNA) corresponds to a $\mathrm{T}$ in $48.5 \%$ of the RNAseq reads. Because the methylation of the $18 \mathrm{~S}$ rRNA affects a nucleotide in close vicinity of the tRNA anticodon at position $\mathrm{P}$ of the ribosome this methylation might be important for proper tRNA positioning and efficient translation. Our results show that methylation of the $18 \mathrm{~S}$ rRNA at this position is conserved in higher plants, and are in line with the hypothesis of the important role of this post-transcription modification in the regulation of plant mitochondrial translation. Surprisingly, both in S. tuberosum and in L. sativa the modification is found in roughly half of the $18 \mathrm{~S}$ molecules, suggesting the existence of two populations of ribosomes.

The mtDNAs of Lactuca species also show differences regarding insertions of exogenous sequences. In the genome of L. saligna LAC008020, a sequence is found that includes a Type B2 superfamily DNA polymerase and a T3/T7 phagetype RNA polymerase ( $d p o, 3,144 \mathrm{bp}$, and rpo, 2,808 bp, on Figure 3). The same sequence was described in the mtDNA of L. saligna CGN5271 (Kozik et al., 2019). These sequences resemble those found in mitochondrial linear plasmids described in other plant species, which at a certain time in evolution physically integrated into the mitochondrial genome (Warren et al., 2016). Interestingly, we identified the same genes in the mtDNA of the three L. virosa accessions ( $d p o, 2,831 \mathrm{bp}$ and rpo, $2,805 \mathrm{bp}$ ). However, only fragments of $r p o$ (319 bp) remain in the L. serriola $\mathrm{mtDNA}$, indicating that these apparently pseudogenes were lost at a certain time during the evolution of Lactuca species. These sequences are no longer found in the mtDNA of L. sativa accessions.

A phylogeny tree was built based on the alignment of the concatenated protein coding sequences, presented in Figure 4A that also includes a table with the number of SNPs and gaps. It shows that there are only $15 \mathrm{SNPs} /$ indels between $L$. sativa and L. virosa mtDNA coding sequences, eightfold less than between L. sativa and L. serriola or L. saligna. Thus, the mtDNA of $L$. sativa is more closely related to the mtDNA of $L$. virosa than to the mitogenomes of the other Lactuca species. As described above, the mtDNA of L. serriola accession US96UC23 is surprisingly identical to the mtDNA of L. sativa.

A second major divergence between the Lactuca mtDNA sequences involves plastid DNA insertions. All genomes share six identical plastid DNA insertions that are from $n d h D$ (194 bp), $\operatorname{pet} G$ (116 bp), petL (81 bp), rbcL (198 bp), rpoB (57 bp), and from $y c f 3(72 \mathrm{bp})$. However, they also have an insertion that is variable between species. The insertion corresponds to a large piece of the plastidial rpoC1 gene $(1,239 \mathrm{bp})$ in $L$. sativa and L. virosa, while in the L. saligna and L. serriola accessions a different insertion is found at the same position, just retaining
$15 \mathrm{bp}$ of the $r p o C 1$ gene but now further comprising $p s a A$ (354 bp) and $p s a B(2,253 \mathrm{bp})$ gene sequences (Figure 4B). The architecture of these two alternative plastidial insertions suggests two independent insertions events during the evolution of the mtDNA. A first one would be the insertion of the large piece of the rpoC1 gene, while a second event, apparently involved recombination between the $r p o C 1$ sequence and another piece of plastidial DNA sharing sequence similarity, resulting in the replacement of most of the $r p o C 1$ sequence by the new insertion. These two different plastidial DNA insertions are interesting markers of Lactuca mtDNA evolution. They further support the closer phylogenetic relationship between the mtDNA genomes of L. sativa and of L. virosa.

The content in repeated sequences also diverges between the different species (Supplementary Table 7). The mtDNA genomes that we assembled contain 17 pairs of repeated sequences larger than 50 bp, in L. sativa and L. virosa, and 18 in L. saligna and L. serriola. All genomes bear a large repeated sequence $(30,534-$ $34,705 \mathrm{bp})$ containing the three rRNA genes. The large pair of repeats $\mathrm{R} 02(10,430 \mathrm{bp})$ is also found in all the mtDNAs that we built. It is extremely conserved and diverges only in L. saligna LAC008020, where it has an insertion of three nucleotides. The two accessions of $L$. serriola show a larger repeat R03 (10 kb) which contains the smaller repeat $\mathrm{R} 03$ found in L. sativa, L. virosa $(3.5 \mathrm{~kb})$ and L. saligna $(4.0 \mathrm{~kb})$. The case of repeat R04 is particular because it is present as three copies in the genomes, two of which are part of the very large repeat R01. It is variable in size in the different genomes (from 576 bp in L. sativa and L. serriola, up to $4,116 \mathrm{pb}$ in L. virosa), but in all species it comprises $320 \mathrm{bp}$ from the $18 \mathrm{~S}$ rRNA gene.

The interpretation of these results is not as clear as the one based on nuclear markers, which show that $L$. sativa and L. serriola are the closest species. On one hand, the loss of $d p o$ and rpo from the mtDNA of both species and the same size of repeated sequence R04 could let us think that the L. sativa mtDNA is closer to the one of L. serriola. But on the other hand, the mtDNA of L. sativa is closer to the one of L. virosa in sequence, by the size of the repeated sequence R03, and by the presence of the rpoC1 plastid insertion.

\section{Lactuca mtDNA Structure Diversity}

To study the structural diversity of these Lactuca mtDNA genomes and the possible events that resulted in their divergence, we aligned them according to the six blocks that subdivide the L. sativa mtDNA (Figure 5A, regions A-F in the representation of the L. sativa mtDNA). Through this alignment it is clear that the mtDNA from L. saligna has the highest structural diversity to L. sativa. The alignment highlighted a recombination event in L. saligna involving an intermediate-size repeated sequence, Rsal of $122 \mathrm{bp}$. The sequence of this repeat can be also found in the mitogenomes of L. sativa, L. serriola, and of L. virosa, but only as a single-copy sequence. We also found that the mtDNAs of $L$. sativa and of $L$. serriola differ in ways that cannot be explained by simple homologous recombination events involving repeated regions. Indeed, as represented in Figure $\mathbf{5 A}$, block C (dark green) in L. serriola has an insertion coming from block B, a genomic rearrangement that we could not find associated with any 

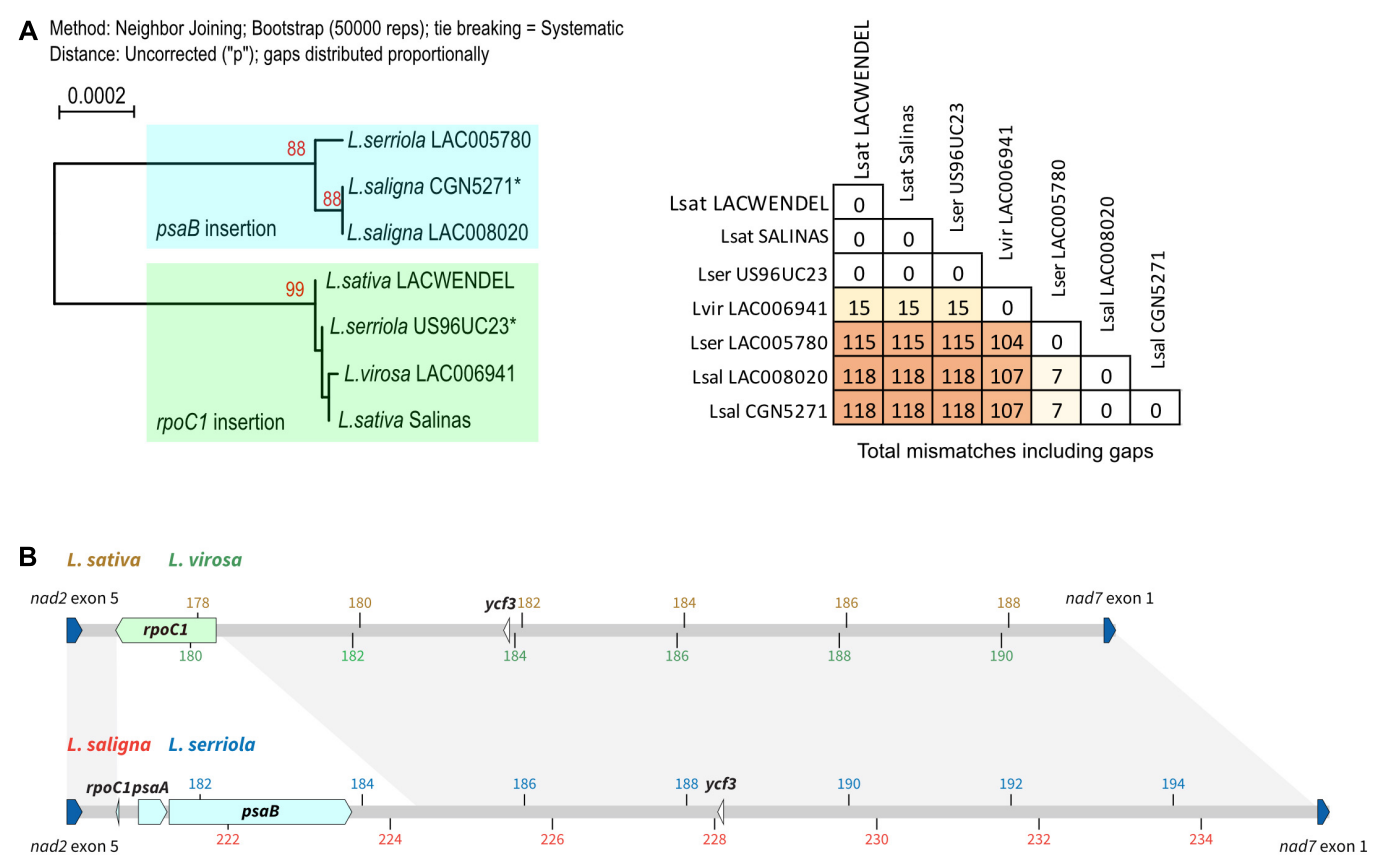

FIGURE 4 | Phylogeny of the mtDNA correlates with different plastidial DNA insertions. (A) Maximum likelihood phylogram based on the alignment of the concatenated protein coding sequences. Bootstrap values are indicated, as well as the table with the number of SNPs and gaps. Sequences labeled with an asterisk (*) are from Kozik et al. (2019). (B) The region between exon 5 of nad2 (last exon of the nad2b transcript) and exon 1 of nad7 (blue boxes) in the mtDNA of L. sativa, L. saligna, L. virosa, and L. serriola are represented with their plastidial DNA insertions (green boxes). Above is shown the context in $L$. sativa and $L$. virosa, with coordinates in brown and green, respectively. Below in L. serriola and L. saligna, with coordinates in blue and red, respectively.

repeated sequence or microhomology. Moreover, as discussed above, L. serriola has a much larger repeat R03 (10,137 bp as compared to $3,553 \mathrm{bp}$ in L. sativa), an extension that came from the $3^{\prime}$ end of the block D. However, these differences between the mtDNAs of the L. serriola accessions that we sequenced and the mtDNA of $L$. sativa are not shared with the recently published mtDNA of L. serriola US96UC23, which is apparently a simple isoform of the L. sativa mtDNA (Figure 5B).

In fact, the mtDNA that is the closest in structure to $L$. sativa is the one of $L$. virosa (Figure $\mathbf{5 A}$ ). Indeed, the only structural divergence between these two mitogenomes is a sequence inversion in the middle of block $\mathrm{F}$, which involves the intermediate-size repeated regions R06 (186 bp). The sequence delimited by this pair of inverted repeats is also larger in L. virosa (30,576 bp long in L. virosa as compared to 21,320 bp in L. sativa). This extension in L. virosa carries the $d p o$ and rpo sequences of apparently viral origin. Consequently it is quite possible that these gene sequences were inserted along other exogenous sequences in the mtDNA by horizontal transfer, explaining the size difference. Thus, in terms of the mitochondrial genome structure, these comparisons show that L. sativa is closer to L. virosa.

\section{Chloroplastic Diversity Among the Lactuca Accessions}

Because the mitogenomes of L. sativa and L. virosa are closely related, in contrast to their nuclear sequences, we considered the possibility that the organellar genomes, which are predominantly maternally inherited, had a different origin. In that case it would be expected that the mitochondrial and plastidial genomes show the same phylogenetic origin. We have therefore tested the chloroplastic diversity among the accessions.

To do so, we build the complete plastidial genome sequences for the four accessions that were sequenced in this study (L. saligna LAC008020, L. sativa LACWendel, L. serriola LAC005780, and L. virosa CGN013357), and for the L. sativa cV. Salinas and L. serriola US96UC23 accessions from which the mtDNAs have been recently published (Kozik et al., 2019). The alignment of these six Lactuca chloroplast genomes, excluding the second copy of the inverted repeat, was 127,738 nucleotides long, comprising 126,686 conserved positions. The polyphyletic tree using Maximum likelihood from this alignment is shown in Figure 6. In this tree, the cpDNA sequences branched as for the nuclear marker (Figure 3). It is possible to distinguish L. saligna and $L$. virosa from $L$. sativa and $L$. serriola, which fall in the same branch of the phylogenetic tree. The number of SNPs and gaps between the cpDNA sequences are also indicated in the figure, showing that there are about eightfold more SNPs/gaps between the cpDNAs of L. sativa and L. virosa than between the cpDNAs of $L$. sativa and $L$. serriola, supporting the conclusion that the cpDNA of $L$. sativa is indeed closer in evolution to the one of L. serriola. Regarding the cpDNA that was assembled from the L. serriola accession US96UC23 raw reads, it is surprisingly virtually identical to the cpDNA genomes of L. sativa. This analysis of the plastidial genome sequences surprisingly suggests divergent origins of the organellar genomes 


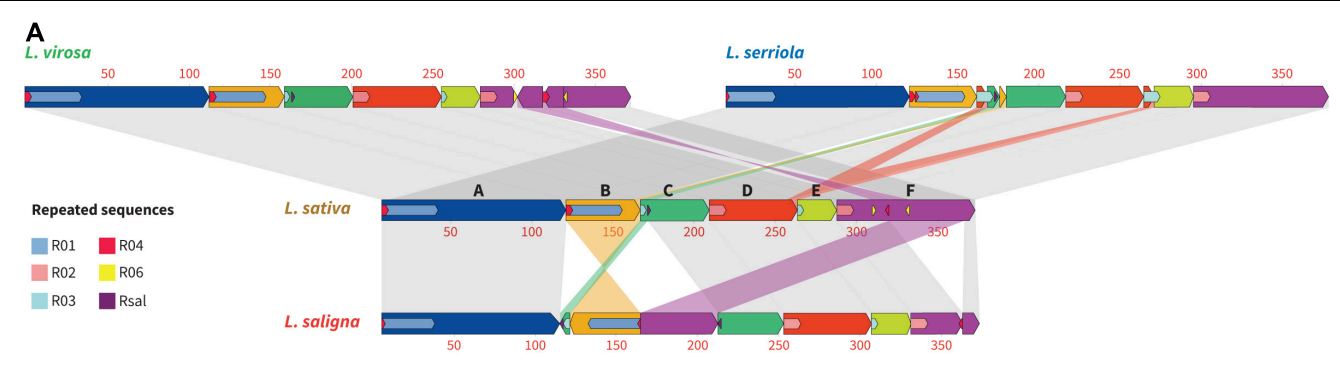

B

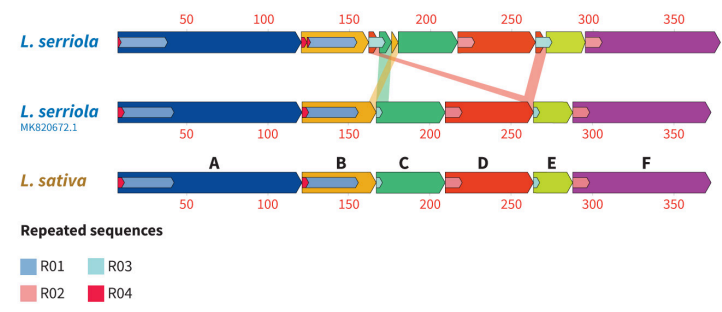

FIGURE 5 | Structural diversity between the mitochondrial genomes of Lactuca spp. Alignments between Lactuca mtDNAs made with progressive Mauve.

(A) Between L. sativa, L. saligna, L. virosa and L. serriola. (B) Between L. sativa, the L. serriola mtDNAs built in our study and the mtDNA of $L$. serriola US96UC23 published by Kozik et al. (2019). Homologous regions delimited by repeated regions are shown in the same color according to the six blocks (A-F) that subdivide the L. sativa mtDNA. Repeated sequences involved in structural evolution between the genomes are represented, according to the key on the left. Scale is in kilobases.

Method: Neighbor Joining; Bootstrap (5000 reps); tie breaking = Systematic Distance: Uncorrected ("p"); Gaps distributed proportionally

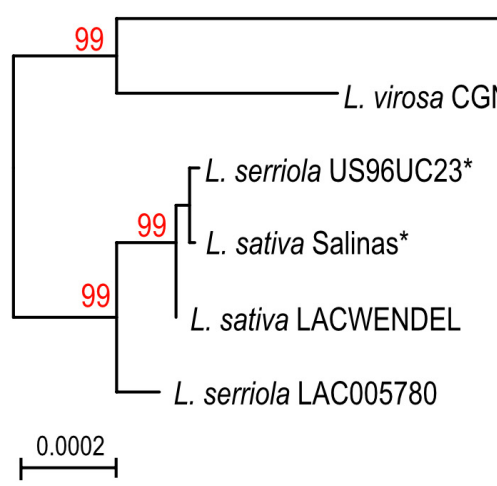

L. saligna LAC008020

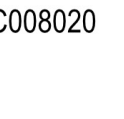

complete cpDNA genomes assembled form the same sequences datasets used to assemble the mitogenomes. Bootstrap values are shown in red and branches
with BS $<80$ are collapsed. Sequences labeled with an asterisk (*) were assembled with raw reads from Kozik et al. (2019), SRX5097892 (L. serriola US96UC23) and SRR577192 (L. sativa CV. Salinas). The table with the number of SNPs and gaps is given.

of L. sativa. The possible causes for such observation are discussed below.

\section{DISCUSSION}

\section{Mitochondrial Diversity Is Low Within Lactuca spp.}

In this study we assembled the mtDNA genomes of nine Lactuca spp. accessions. Our assembly of the mtDNA of $L$. sativa
Butterhead lettuce (var. capitata L. nidus tenerrima) is robust, based on Illumina short reads and PacBio long reads, which allowed us to infer one of the major isoform structures of the mtDNA. During this work the mtDNA of a Crisphead lettuce accession was published by Kozik et al. (2019), corresponding to the same genome in a different isomeric form. The concordance of the two independent results validated our assembly. We further built eight additional mtDNA genomes of other accessions and species, from Illumina sequence data obtained from purified mitochondria. The assemblies were based on the possible order 
of scaffolds inferred from overlapping sequences. We validated our assembly pipeline on L. sativa, because from just Illumina sequence data we obtained the same genome structure as the one assembled with the help of PacBio long reads. Our assembly of the mtDNA of L. saligna LAC008020 further supported the validity of our assembly pipeline, because the structure we inferred is a perfect isoform to the published mtDNA of L. saligna CGN5271 (Kozik et al., 2019). We are therefore confident that the structures we present are valid, and correspond to one of the major isoforms of the mtDNA, interconvertible to other possible ones by recombination involving the large repeated sequences.

Unlike what we expected, we found very little mitochondrial diversity at both the interspecies and intraspecies levels in this panel. This is surprising, when considering the very distant geographical origins of the accessions (Supplementary Table 1). But the geographical origins might just reflect the recent humanmediated dispersal of the species, distorting the picture of their natural geographical range and of the origin of the domesticated species. Indeed, at the intraspecies level we found no differences between the mitogenomes of all $L$. sativa accessions analyzed, although they belong to different typologies, nor among the three $L$. virosa accessions, or between the two L. serriola accessions we sequenced. The mtDNA of the L. saligna we sequenced and assembled is also identical to the one of the L. saligna accession published by Kozik et al. (2019). This is in contrast with what was found in other species, such as Arabidopsis, Brassica napus or maize, in which the mitogenomes from different subspecies or even different accessions can vary significantly in organization. We did not expect major differences between species with respect to the mtDNA coding content, because the content of plant mitogenomes varies barely interspecies and differences are further reduced when comparing evolutionary close species. The gene sequences were also expected to be highly conserved, because apart from some notorious genus such as Silene and Plantago (Mower et al., 2007; Sloan et al., 2012), the sequences of higher plant mitochondrial genes evolve very slowly (Palmer and Herbon, 1988). Still, apart from the re-organization of genomic regions, we found very few SNPs or indels between the mitogenomes of the Lactuca species we studied. The little mitochondrial diversity at the interspecies and intraspecies levels indicates recent divergence of these species and accessions. However, a demographic history inference based on nuclear SNPs suggested that cultivated lettuce likely originated from the Fertile Crescent more than 12,000 years ago (Zhang et al., 2017). And while L. saligna and L. serriola have geographical origins overlapping with the presumed origin of domesticated L. sativa, which is South-West Asia, L. virosa has a distinct geographical origin (Kesseli et al., 1991).

\section{Divergence of mtDNA Sequences and Structures Among L. serriola Accessions}

The major intraspecies differences we found concerned the mtDNA of the L. serriola accessions we assembled (CGN004799 and LAC005780) and the one of L. serriola US96UC23 that was published by Kozik et al. (2019). Its structure is very different from the ones of CGN004799 and LAC005780 (Figure 5B). It is also different in sequence, as the mitogenomes of CGN004799 and LAC005780 carry one additional copy of the $\operatorname{trnD}(\mathrm{GUC})$ gene of plastidial origin, which is absent in US96UC23 (Supplementary Table 6). Most significant, the mtDNA of L. serriola US96UC23 carries the same plastidial rpoC1 insertion that is found in the mtDNAs of L. sativa and of L. virosa, while at the same position the mitogenomes of the two other $L$. serriola accessions contain instead the psaA-psaB insertion that is also found in the mtDNA of L. saligna. Moreover, the mitogenome of L. serriola US96UC23 does not contain any remaining sequences of the dpo-rpo insertion, like in L. sativa. Thus, the structure and sequence of the mitogenome of L. serriola US96UC23 is very different from the mtDNAs of CGN004799 and LAC005780 and rather looks like the mtDNA of L. sativa (Figure 5B). These observations and their implications are further discussed below.

\section{Analysis of the Lettuce Mitogenomes Suggest an Important Role of $L$. virosa in Lettuce Diversification}

The involvement of the different Lactuca species in the domestication and/or diversification of $L$. sativa is not clearly understood. From a nuclear genome point of view L. serriola is considered as one of the direct ancestors and the closest relative to L. sativa (de Vries, 1990; de Vries and van Raamsdonk, 1994). A recent study based on nuclear RNA-seq analysis further supported the hypothesis that all typologies of cultivated lettuce originated from a single domestication event and a common ancestor, which was a wild $L$. serriola or an already domesticated one (Zhang et al., 2017). Further diversification of L. sativa would have led first to the Butterhead typology and later to Crisphead lettuces (Zhang et al., 2017). Our results on the nuclear and chloroplastic sequence diversity among our accessions are in line with this assumption. Indeed, according to these results, L. saligna and $L$. virosa are distant from the pair L. serriola- $L$. sativa. Consequently, the role of the others Lactuca spp. seemed limited to the development of new lettuce cultivars (Zhang et al., 2017). Indeed, intraspecies crosses have been used by breeders for the development of new cultivars, and a relevant example is found in the genealogy of North American lettuces, which showed that current Crisphead cultivars can be split into two groups, one derived from cultivars where L. virosa was introgressed in L. sativa to breed for robust root system and decreased leaf drop, and a second group that is not $L$. virosa derived (Mikel, 2007). But besides the development of current cultivars, no involvement of $L$. virosa on Lettuce domestication has been described, and was also considered unlikely because it has a different geographical center of origin than L. serriola and L. sativa (Lebeda et al., 2004).

In this work, we have for the first time assembled mitogenomes from $L$. virosa. We have also built the mtDNA of three additional L. sativa accessions. Among these, two (LAC004500 and UC12100) are established and documented as Crisphead lettuce, respectively, while the third (LACWendel) is a Butterhead lettuce. Unexpectedly, both in sequence and structure we found higher similarity between the mtDNAs of $L$. virosa and L. sativa, including Crisphead and Butterhead lettuces, than with L. serriola 
and L. saligna. Most significantly, the L. virosa and L. sativa mitogenomes share the same chloroplastic insertion, while a different one is found at the same locus in L. serriola and L. saligna. The possibility that a same event of horizontal transfer happened twice in evolution is unrealistic. Moreover, the alignment of the concatenated mtDNA protein coding sequences supported that the L. sativa mtDNA is closer to the one of L. virosa than to the ones of L. serriola (CGN004799 and LAC005780) or L. saligna (Figure 4A).

This closeness between the mtDNAs of L. virosa and L. sativa, of both Crisphead and Butterhead typologies, suggests that cultivated lettuce might be derived from an ancestral cross where L. virosa was the female partner that transmitted its mitogenome, and that consequently $L$. virosa played a more important role in L. sativa domestication than previously suspected. Backcrosses would have cleaned the actual $L$. sativa nuclear genome from most L. virosa sequences, as observed at present (Zhang et al., 2017). However, this scenario does not explain the heterogeneity of mitogenomes observed among $L$. serriola accessions, with the one of $L$. serriola US96UC23 almost identical to the one of $L$. sativa. The cpDNA genome assembled from the same Illumina sequences data set is also virtually identical to the cpDNA of L. sativa. This observation calls for the hypothesis that this sequence data set originates from a $L$. sativa accession mislabeled as L. serriola. An alternative possibility is that L. virosa had previously been hybridized with $L$. serriola, leading to two different $L$. serriola groups; a wild one harboring a mtDNA distinct from the one of $L$. virosa and a second group derived from the hybrid, which was later the precursor of cultivated L. sativa.

\section{The Divergence Between cpDNA and mtDNA Suggests Paternal Leakage}

The hypothesis according to which $L$. virosa was introgressed as a cytoplasm donor during L. sativa diversification can explain why the mitogenomes of the two species are so similar. However, it does not fit with the phylogeny based on the chloroplast genomes alignment. Indeed, while the mtDNA of $L$. sativa is closer to the one of L. virosa, its cpDNA is closer to the cpDNA of L. serriola. In most flowering plant species, lettuces included, the mtDNA and cpDNA are both maternally transmitted and should follow the same inheritance. The apparent divergent origins of the organellar genomes could be because one was transmitted paternally by paternal leakage, meaning that during a cross organelles and their genomes were transmitted via the pollen.

Plastids and the cpDNA are maternally inherited due to the degeneration/exclusion of plastids from the pollen generative cell, or because elimination of paternal plastids during fertilization (Hagemann and Schröder, 1989; Mogensen, 1996). But a lowfrequency leakage of plastids is commonly admitted in plants. In $A$. thaliana, plastids are absent in pollen sperm cells and consequently only maternally inherited (Martínez et al., 1997; Nagata et al., 1999). The frequency of paternal plastid transmission was evaluated as low as 1.9-3.9 $\times 10^{-5}$ (Azhagiri and Maliga, 2007; Schneider et al., 2015). Other studies described similar low frequencies in other species, like in Setaria italica,

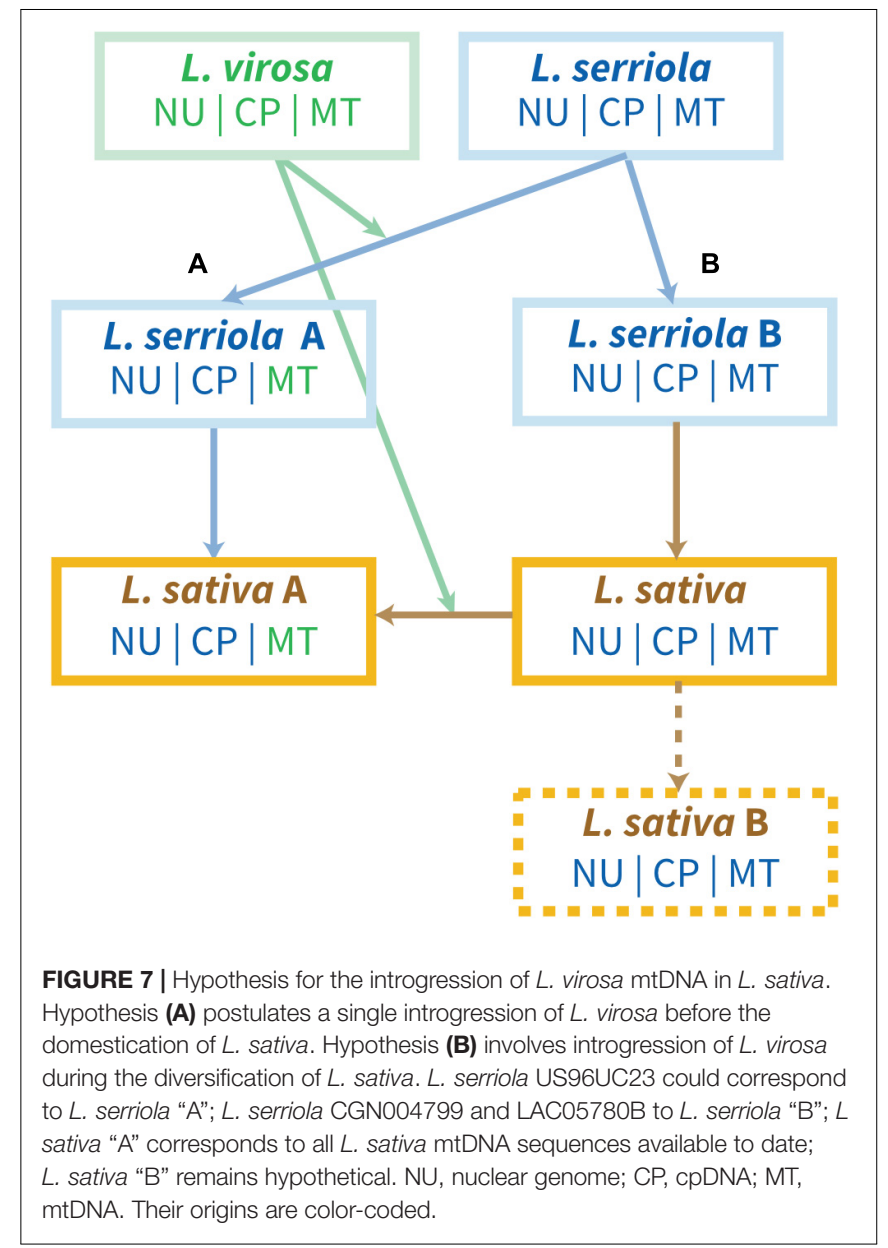

Brassica napus, and Antirrhinum majus (Diers, 1967; Wang et al., 2004; Schneider et al., 2015). However, because plastid diversity follows nuclear diversity within the Lactuca population, with the couple L. sativa/L. serriola distinct from the couple L. saligna/L. virosa, it appears that if paternal leakage did indeed occur, it involved the mitochondrial genome.

Mitochondrial paternal leakage events have been highlighted in natural populations and linked with heteroplasmy in Silene acaulis (Delph et al., 2007), Silene vulgaris (Wade and McCauley, 2005) or in Daucus carota ssp. carota, a weedy relative to domesticated carrot (Mandel et al., 2012). Preferential paternal transmission of the mitochondrial genome has been described in melon (Cucumis melo) and cucumber (Cucumis sativus), with rare maternal and biparental transmission (Havey et al., 1998; Shen et al., 2019). But there is no evidence of paternal mtDNA transmission in Lactuca spp. If we agree on an ancestral event of mitochondrial paternal leakage within the Lactuca population, and considering the heterogeneity of the mitogenomes among L. serriola accessions, we can consider an alternative hypothesis to explain the role of $L$. virosa in the evolution of the L. sativa mitogenome (Figure 7). This hypothesis relies on introgression of $L$. virosa either before the domestication of $L$. sativa, during a cross with an ancestral $L$. serriola, or later during the diversification of L. sativa, which would have led to mitochondrial 
paternal leakage. Paternal leakage would result in heteroplasmic mtDNA in the cell, and it has been shown that the coexistence of two mitogenomes results in recombination and sorting of a new predominant mtDNA containing pieces of the original genomes (Sanchez-Puerta et al., 2015). That could explain why the L. sativa mtDNA has certain regions that seem to be closer to L. serriola, like the loss of $d p o$ and rpo and the size of repeated sequence R04, and other regions that are clearly related to the mtDNA of $L$. virosa, such as the cpDNA insertion. Later, the progeny of this hybridization, carrying nuclear and cpDNA genomes still related to $L$. serriola and an mtDNA mostly derived from $L$. virosa would have been used in L. sativa domestication. A future analysis of the mtDNA variability among Asteraceae should support or disprove this hypothesis. Our present study was restricted to a few accessions of a just a few species of the genus Lactuca, the ones most often used in crosses for the diversification of L. sativa, and a more comprehensive collection of Lactuca species is needed to achieve a clearer picture of the evolution of their mitogenomes. The study of a broader collection of L. sativa accessions covering all know typologies would also help to shed light on when its mtDNA diverged from the one of its L. serriola ancestor. Extraction of the mtDNA sequences from the recently described data set of 445 accessions (Wei et al., 2021) seems to be an obvious next step.

\section{DATA AVAILABILITY STATEMENT}

The datasets presented in this study can be found in online repositories. The names of the repository/repositories and accession number(s) can be found below: https://www.ncbi.nlm. nih.gov/genbank/, MZ159953; https:/www.ncbi.nlm.nih.gov/ genbank/, MZ159954; https://www.ncbi.nlm.nih.gov/genbank/, MZ159955; https://www.ncbi.nlm.nih.gov/genbank/, MZ159956; https://www.ncbi.nlm.nih.gov/genbank/, MZ159957; https:// www.ncbi.nlm.nih.gov/genbank/, MZ159958; https://www.ncbi. nlm.nih.gov/genbank/, MZ159959; https://www.ncbi.nlm.nih. gov/genbank/, MZ159960; https://www.ncbi.nlm.nih.gov/ genbank/, MZ159961; https://www.ncbi.nlm.nih.gov/genbank/,

\section{REFERENCES}

Altschul, S. F., Gish, W., Miller, W., Myers, E. W., and Lipman, D. J. (1990). Basic local alignment search tool. J. Mol. Biol. 215, 403-410.

Azhagiri, A. K., and Maliga, P. (2007). Exceptional paternal inheritance of plastids in Arabidopsis suggests that low-frequency leakage of plastids via pollen may be universal in plants. Plant J. 52, 817-823. doi: 10.1111/j.1365-313x.2007.03278.x

Bankevich, A., Nurk, S., Antipov, D., Gurevich, A. A., Dvorkin, M., Kulikov, A. S., et al. (2012). SPAdes: a new genome assembly algorithm and its applications to single-cell sequencing. J. Comput. Biol. 19, 455-477. doi: 10.1089/cmb.2012. 0021

Bennett, M. D., Leitch, I. J., Price, H. J., and Johnston, J. S. (2003). Comparisons with Caenorhabditis (approximately $100 \mathrm{Mb}$ ) and Drosophila (approximately $175 \mathrm{Mb}$ ) using flow cytometry show genome size in Arabidopsis to be approximately $157 \mathrm{Mb}$ and thus approximately 25\% larger than the Arabidopsis genome initiative estimate of approximately $125 \mathrm{Mb}$. Ann. Bot. 91, 547-557. doi: $10.1093 / \mathrm{aob} / \mathrm{mcg} 057$
ERS5267070; https://www.ncbi.nlm.nih.gov/genbank/, ERS 5267071; https://www.ncbi.nlm.nih.gov/genbank/, ERS 5267072; https://www.ncbi.nlm.nih.gov/genbank/, ERS5267073; https://www.ncbi.nlm.nih.gov/genbank/, ERS5267074; https:// www.ncbi.nlm.nih.gov/genbank/, ERS5267075; https://www.ncbi. nlm.nih.gov/genbank/, ERS5267076; https://www.ncbi.nlm.nih. gov/genbank/, ERS5267077; and https://www.ncbi.nlm.nih.gov/ genbank/, ERS5267078.

\section{AUTHOR CONTRIBUTIONS}

AF, EG-F, G-JB, and JG designed the experiments. AF, SK, and EG-F performed the experimental work. AF and SG processed the sequence data. AF and JG analyzed the data and wrote the manuscript with contribution of all co-authors.

\section{FUNDING}

AF received a Ph.D. scholarship through the French CIFRE program (2016/1154). Research in the lab of JG benefits from funding managed by the French National Research Agency as part of the "Investments for the future" program (ANR-11-LABX0057_MITOCROSS).

\section{ACKNOWLEDGMENTS}

We acknowledge support from all members of the JMF Laboratory, and of the technical platforms of the IBMP. The help of Sébastien Staerck for the growth of plants is particularly recognized.

\section{SUPPLEMENTARY MATERIAL}

The Supplementary Material for this article can be found online at: https://www.frontiersin.org/articles/10.3389/fpls.2021. 697136/full\#supplementary-material

Bergthorsson, U., Adams, K. L., Thomason, B., and Palmer, J. D. (2003). Widespread horizontal transfer of mitochondrial genes in flowering plants. Nature 424, 197-201. doi: 10.1038/nature01743

Bolger, A. M., Lohse, M., and Usadel, B. (2014). Trimmomatic: a flexible trimmer for Illumina sequence data. Bioinformatics 30, 2114-2120. doi: 10.1093/ bioinformatics/btu170

Cognat, V., Morelle, G., Megel, C., Lalande, S., Molinier, J., Vincent, T., et al. (2017). The nuclear and organellar tRNA-derived RNA fragment population in Arabidopsis thaliana is highly dynamic. Nucleic Acids Res. 45, 3460-3472. doi: 10.1093/nar/gkw1122

Cummings, M. P., Nugent, J. M., Olmstead, R. G., and Palmer, J. D. (2003). Phylogenetic analysis reveals five independent transfers of the chloroplast gene rbcL to the mitochondrial genome in angiosperms. Curr. Genet. 43, 131-138. doi: 10.1007/s00294-003-0378-3

Darling, A. E., Mau, B., and Perna, N. T. (2010). progressiveMauve: multiple genome alignment with gene gain, loss and rearrangement. PLoS One 5:e11147. doi: 10.1371/journal.pone.0011147 
Davila, J. I., Arrieta-Montiel, M. P., Wamboldt, Y., Cao, J., Hagmann, J., Shedge, V., et al. (2011). Double-strand break repair processes drive evolution of the mitochondrial genome in Arabidopsis. BMC Biol. 9:64. doi: 10.1186/17417007-9-64

Davis, R. M., Subbarao, K. V., Raid, R. N., and Kurtz, A. K. (1997). Compendium of Lettuce Diseases. Eagan, MN: APS Press.

de Vries, I. M. (1990). Crossing experiments of lettuce cultivars and species (Lactuca sect.Lactuca, Compositae). Plant Syst. Evol. 171, 233-248. doi: 10. 1007/bf00940608

de Vries, I. M., and van Raamsdonk, L. W. D. (1994). Numerical morphological analysis of Lettuce cultivars and species (Lactuca sect.Lactuca, Asteraceae). Plant Syst. Evol. 193, 125-141. doi: 10.1007/bf00983546

Delph, L., Touzet, P., and Bailey, M. (2007). Merging theory and mechanism in studies of gynodioecy. Trends Ecol. Evol. 22, 17-24. doi: 10.1016/j.tree.2006.09. 013

Dierckxsens, N., Mardulyn, P., and Smits, G. (2016). NOVOPlasty: de novo assembly of organelle genomes from whole genome data. Nucleic Acids Res. 45:e18.

Diers, L. (1967). Übertragung von Plastiden durch den Pollen bei Antirrhinum majus. Mol. Gen. Genet. 100, 56-62. doi: 10.1007/bf00425775

Dietrich, A., Maréchal-Drouard, L., Carneiro, V., Cosset, A., and Small, I. (1996). A single base change prevents import of cytosolic tRNAAla into mitochondria in transgenic plants. Plant J. 10, 913-918. doi: 10.1046/j.1365-313x.1996. 10050913.x

Edgar, R. C. (2004). MUSCLE: multiple sequence alignment with high accuracy and high throughput. Nucleic Acids Res. 32, 1792-1797. doi: 10.1093/nar/gkh340

Garcia, L. E., Zubko, M. K., Zubko, E. I., and Sanchez-Puerta, M. V. (2019). Elucidating genomic patterns and recombination events in plant cybrid mitochondria. Plant Mol. Biol. 100, 433-450. doi: 10.1007/s11103-01900869-z

Garrison, E., and Marth, G. (2012). Haplotype-based variant detection from shortread sequencing. ArXiv 1207.3907v2. Available online at: https://arxiv.org/abs/ $1207.3907 \mathrm{v} 2$

Hagemann, R., and Schröder, M. B. (1989). The cytological basis of the plastid inheritance in angiosperms. Protoplasma 152, 57-64. doi: 10.1007/BF0132 3062

Havey, M. J., McCreight, J. D., Rhodes, B., and Taurick, G. (1998). Differential transmission of the Cucumis organellar genomes. Theor. Appl. Genet. 97, 122-128. doi: 10.1007/s001220050875

Kesseli, R., Ochoa, O., and Michelmore, R. (1991). Variation at RFLP loci in Lactuca spp. and origin of cultivated lettuce (L . sativa). Genome 34, 430-436. doi: 10.1139/g91-065

Kim, D., Langmead, B., and Salzberg, S. L. (2015). HISAT: a fast spliced aligner with low memory requirements. Nat. Methods 12, 357-360. doi: 10.1038/nmeth. 3317

Koopman, W. J. M., Guetta, E., van de Wiel, C. C. M., Vosman, B., and van den Berg, R. G. (1998). Phylogenetic relationships among Lactuca (Asteraceae) species and related genera based on ITS-1 DNA sequences. Am. J. Bot. 85, 1517-1530. doi: 10.2307/2446479

Koopman, W. J. M., Zevenbergen, M. J., and Van den Berg, R. G. (2001). Species relationships in Lactuca s.l. (Lactuceae, Asteraceae) inferred from AFLP fingerprints. Am. J. Bot. 88, 1881-1887. doi: 10.2307/ 3558364

Koren, S., Walenz, B. P., Berlin, K., Miller, J. R., Bergman, N. H., and Phillippy, A. M. (2017). Canu: scalable and accurate long-read assembly via adaptive $k$ mer weighting and repeat separation. Genome Res. 27, 722-736. doi: 10.1101/ gr.215087.116

Kozik, A., Rowan, B. A., Lavelle, D., Berke, L., Schranz, M. E., Michelmore, R. W., et al. (2019). The alternative reality of plant mitochondrial DNA: one ring does not rule them all. PLoS Genet. 15:e1008373. doi: 10.1371/journal.pgen.1008373

Kumar, S., Stecher, G., Li, M., Knyaz, C., and Tamura, K. (2018). MEGA X: molecular evolutionary genetics analysis across computing platforms. Mol. Biol. Evol. 35, 1547-1549. doi: 10.1093/molbev/msy096

Langmead, B., Trapnell, C., Pop, M., and Salzberg, S. L. (2009). Ultrafast and memory-efficient alignment of short DNA sequences to the human genome. Genome Biol. 10:R25.
Lebeda, A., Dolezalová, I., Feráková, V., and Astley, D. (2004). Geographical distribution of wild lactuca species (Asteraceae, Lactuceae). Bot. Rev. 70, 328356. doi: 10.1663/0006-8101(2004)070[0328:gdowls]2.0.co;2

Letunic, I., and Bork, P. (2019). Interactive Tree Of Life (iTOL) v4: recent updates and new developments. Nucleic Acids Res. 47, W256-W259.

Li, H., and Durbin, R. (2009). Fast and accurate short read alignment with BurrowsWheeler transform. Bioinformatics 25, 1754-1760. doi: 10.1093/bioinformatics/ btp324

Li, H., Handsaker, B., Wysoker, A., Fennell, T., Ruan, J., Homer, N., et al. (2009). The sequence Alignment/Map format and SAMtools. Bioinformatics 25, 20782079. doi: 10.1093/bioinformatics/btp352

Lindqvist, K. (1960). On the origin of cultivated lettuce. Hereditas 46, 319-350. doi: 10.1111/j.1601-5223.1960.tb03091.x

Mandel, J. R., McAssey, E. V., Roland, K. M., and McCauley, D. E. (2012). Mitochondrial gene diversity associated with the atp9 stop codon in natural populations of wild carrot (Daucus carota ssp. carota). J. Hered. 103, 418-425. doi: 10.1093/jhered/esr142

Marienfeld, J., Unseld, M., and Brennicke, A. (1999). The mitochondrial genome of Arabidopsis is composed of both native and immigrant information. Trends Plant Sci. 4, 495-502. doi: 10.1016/s1360-1385(99)01502-2

Martínez, P., López, C., Roldán, M., Sabater, B., and Martín, M. (1997). Plastid DNA of five ecotypes of Arabidopsis thaliana: sequence of ndhG gene and maternal inheritance. Plant Sci. 123, 113-122. doi: 10.1016/s0168-9452(96)04563-3

Mikel, M. A. (2007). Genealogy of contemporary north american lettuce. HortScience 42, 489-493. doi: 10.21273/hortsci.42.3.489

Mogensen, H. L. (1996). The hows and whys of cytoplasmic inheritance in seed plants. Amer. J. Bot. 83, 383-404.

Mower, J. P., Touzet, P., Gummow, J. S., Delph, L. F., and Palmer, J. D. (2007). Extensive variation in synonymous substitution rates in mitochondrial genes of seed plants. BMC Evol. Biol. 7:135. doi: 10.1186/1471-2148-7-135

Nagata, N., Saito, C., Sakai, A., Kuroiwa, H., and Kuroiwa, T. (1999). The selective increase or decrease of organellar DNA in generative cells just after pollen mitosis one controls cytoplasmic inheritance. Planta 209, 53-65. doi: 10.1007/ s004250050606

Newton, K. J., Mariano, J. M., Gibson, C. M., Kuzmin, E., and Gabay-Laughnan, S. (1996). Involvement of S2 episomal sequences in the generation of NCS4 deletion mutation in maize mitochondria. Dev. Genet. 19, 277-286. doi: 10. 1002/(sici)1520-6408(1996)19:3<277::aid-dvg11>3.0.co;2-x

Palmer, J. D., and Herbon, L. A. (1988). Plant mitochondrial DNA evolved rapidly in structure, but slowly in sequence. J. Mol. Evol. 28, 87-97. doi: 10.1007/ bf02143500

Parra, L., Nortman, K., Sah, A., Truco, M. J., Ochoa, O., and Michelmore, R. (2021). Identification and mapping of new genes for resistance to downy mildew in lettuce. Theor. Appl. Genet. 134, 519-528. doi: 10.1007/s00122-020-03711-z

Reyes-Chin-Wo, S., Wang, Z., Yang, X., Kozik, A., Arikit, S., Song, C., et al. (2017). Genome assembly with in vitro proximity ligation data and whole-genome triplication in lettuce. Nat. Commun. 8:14953.

Ryder, E. J. (1999). Lettuce, Endive and Chicory. Wallingford: CABI Publishing.

Robinson, J. T., Thorvaldsdóttir, H., Winckler, W., Guttman, M., Lander, E. S., Getz, G., et al. (2011). Integrative genomics viewer. Nat. Biotechnol. 29, 24-26. doi: $10.1038 /$ nbt.1754

Sanchez-Puerta, M. V., Zubko, M. K., and Palmer, J. D. (2015). Homologous recombination and retention of a single form of most genes shape the highly chimeric mitochondrial genome of a cybrid plant. New Phytol. 206, 381-396. doi: $10.1111 / \mathrm{nph} .13188$

Schneider, A., Stelljes, C., Adams, C., Kirchner, S., Burkhard, G., Jarzombski, S., et al. (2015). Low frequency paternal transmission of plastid genes in Brassicaceae. Transgenic Res. 24, 267-277. doi: 10.1007/s11248-014-9842-8

Shen, J., Shou, W., Zhang, Y., Yuan, G., Zhao, Y., Chen, J., et al. (2019). Rare maternal and biparental transmission of the cucumber mitochondrial DNA reveals sorting of polymorphisms among progenies. Theor. Appl. Genet. 132, 1223-1233. doi: 10.1007/s00122-018-03274-0

Sloan, D. B., Alverson, A. J., Chuckalovcak, J. P., Wu, M., McCauley, D. E., Palmer, J. D., et al. (2012). Rapid evolution of enormous, multichromosomal genomes in flowering plant mitochondria with exceptionally high mutation rates. PLoS Biol. 10:e1001241. doi: 10.1371/journal.pbio.1001241 
Sloan, D. B., Wu, Z., and Sharbrough, J. (2018). Correction of persistent errors in arabidopsis reference mitochondrial genomes. Plant Cell 30, 525-527. doi: $10.1105 /$ tpc. 18.00024

Stecher, G., Tamura, K., and Kumar, S. (2020). Molecular Evolutionary Genetics Analysis (MEGA) for macOS. Mol. Biol. Evol. 37, 1237-1239. doi: 10.1093/ molbev/msz312

Takemura, M., Oda, K., Yamato, K., Ohta, E., Nakamura, Y., Nozato, N., et al. (1992). Gene clusters for ribosomal proteins in the mitochondrial genome of a liverwort, Marchantia polymorpha. Nucleic Acids Res. 20, 3199-3205. doi: 10.1093/nar/20.12.3199

The Arabidopsis Genome Initiative. (2000). Analysis of the genome sequence of the flowering plant Arabidopsis thaliana. Nature 408, 796-815. doi: 10.1038/ 35048692

Thompson, R. C. (1943). Futher studies on interspecific genetic relationships in Lactuca. J. Agric. Res. 66, 41-48.

Thompson, R. C., Whitaker, T. W., and Kosar, W. F. (1941). Interspecific genetic relationships in Lactuca. J. Agric. Res. 63, 91-107.

Tillich, M., Lehwark, P., Pellizzer, T., Ulbricht-Jones, E. S., Fischer, A., Bock, R., et al. (2017). GeSeq - versatile and accurate annotation of organelle genomes. Nucleic Acids Res. 45, W6-W11.

Varré, J. S., D’Agostino, N., Touzet, P., Gallina, S., Tamburino, R., Cantarella, C., et al. (2019). Complete sequence, multichromosomal architecture and transcriptome analysis of the solanum tuberosum mitochondrial genome. Int. J. Mol. Sci. 20:4788. doi: 10.3390/ijms20194 788

Wade, M. J., and McCauley, D. E. (2005). Paternal leakage sustains the cytoplasmic polymorphism underlying gynodioecy but remains invasible by nuclear restorers. Am. Nat. 166, 592-602. doi: 10.1086/491660

Waltz, F., Soufari, H., Bochler, A., Giegé, P., and Hashem, Y. (2020). CryoEM structure of the RNA-rich plant mitochondrial ribosome. Nat. Plants 6, 377-383. doi: 10.1038/s41477-020-0631-5

Wang, T., Li, Y., Shi, Y., Reboud, X., Darmency, H., and Gressel, J. (2004). Low frequency transmission of a plastid-encoded trait in Setaria italica. Theor. Appl. Genet. 108, 315-320. doi: 10.1007/s00122-003-1424-8

Warren, J. M., Simmons, M. P., Wu, Z., and Sloan, D. B. (2016). Linear plasmids and the rate of sequence evolution in plant mitochondrial genomes. Genome Biol. Evol. 8, 364-374. doi: 10.1093/gbe/evw003
Wei, T., van Treuren, R., Liu, X., Zhang, Z., Chen, J., Liu, Y., et al. (2021). Wholegenome resequencing of 445 Lactuca accessions reveals the domestication history of cultivated lettuce. Nat. Genet. 53, 752-760. doi: 10.1038/s41588-02100831-0

Wei, Z., Zhu, S.-X., Van den Berg, R. G., Bakker, F. T., and Schranz, M. E. (2017) Phylogenetic relationships within Lactuca L. (Asteraceae), including African species, based on chloroplast DNA sequence comparisons. Genet. Resour. Crop Evol. 64, 55-71. doi: 10.1007/s10722-015-0332-5

Wynn, E. L., and Christensen, A. C. (2019). Repeats of unusual size in plant mitochondrial genomes: identification, incidence and evolution. G3-Genes Genomes Genet. 9, 549-559. doi: 10.1534/g3.118.200948

Zerbino, D. R., and Birney, E. (2008). Velvet: algorithms for de novo short read assembly using de Bruijn graphs. Genome Res. 18, 821-829. doi: 10.1101/gr. 074492.107

Zhang, L., Su, W., Tao, R., Zhang, W., Chen, J., Wu, P., et al. (2017). RNA sequencing provides insights into the evolution of lettuce and the regulation of flavonoid biosynthesis. Nat. Commun. 8:2264.

Conflict of Interest: G-JB and EG-F were employed by the company Enza Zaden.

The remaining authors declare that the research was conducted in the absence of any commercial or financial relationships that could be construed as a potential conflict of interest.

Publisher's Note: All claims expressed in this article are solely those of the authors and do not necessarily represent those of their affiliated organizations, or those of the publisher, the editors and the reviewers. Any product that may be evaluated in this article, or claim that may be made by its manufacturer, is not guaranteed or endorsed by the publisher.

Copyright (C) 2021 Fertet, Graindorge, Koechler, de Boer, Guilloteau-Fonteny and Gualberto. This is an open-access article distributed under the terms of the Creative Commons Attribution License (CC BY). The use, distribution or reproduction in other forums is permitted, provided the original author(s) and the copyright owner(s) are credited and that the original publication in this journal is cited, in accordance with accepted academic practice. No use, distribution or reproduction is permitted which does not comply with these terms. 